\title{
Effect of Amphiphile Hydrophobicity on Wetting Behaviors of Ternary Water + Oil + Amphiphile Mixtures: A Density Functional Theory Approach
}

\author{
Ming-Chih Yeh, Chia-Ming Chen, and Li-Jen Chen* \\ Department of Chemical Engineering, National Taiwan University, Taipei, Taiwan 10617, Republic of China
}

Received: November 30, 2003; In Final Form: March 26, 2004

\begin{abstract}
In this study, the statistical associating fluid theory (SAFT) combined with the density functional theory (DFT) was applied to explore the phase and wetting behavior of ternary water + oil + amphiphile mixtures. It is well understood that this type of mixture exhibits a three-liquid-phase coexisting under the condition of certain temperature, pressure and concentration ranges. The Helmholtz free energy model of SAFT successfully delineates the phase behavior of the water + oil + amphiphile ternary mixture. The DFT was further applied to calculate the interfacial tensions of the coexisting phases. The wetting behavior of the amphiphile-rich phase at the interface separating the oil-rich and aqueous phases was then determined according to whether the interfacial tensions obey either Antonow's rule or Neumann inequality. The occurrence of wetting transitions was systematically explored while the system is brought to its critical end point. A parameter $R$ was proposed to emulate the amphiphile hydrophobicity. A sequence of wetting transitions, nonwetting $\rightarrow$ partial-wetting $\rightarrow$ complete-wetting, along with increasing temperature was found by simply varying $R$. These predictions are consistent with the experimental observations on wetting behaviors of ternary water + oil $+\mathrm{C}_{i} \mathrm{E}_{j}$ mixtures, where $\mathrm{C}_{i} \mathrm{E}_{j}$ is the abbreviation of a nonionic surfactant polyoxyethylene alcohol $\mathrm{C}_{i} \mathrm{H}_{2 i+1}\left(\mathrm{OCH}_{2} \mathrm{CH}_{2}\right)_{j} \mathrm{OH}$. The order of wetting transitions is also discussed.
\end{abstract}

\section{Introduction}

Consider a system of three phases $\alpha, \beta$, and $\gamma$, in equilibrium under gravity. The densities of these three phases are in the order $\rho_{\alpha}>\rho_{\beta}>\rho_{\gamma}$. The wetting behavior of the middle $\beta$ phase can be classified into three groups according to the relationship of interfacial tensions as shown in Figure $1:^{1}$ (a) complete wetting, (b) partial wetting, and (c) nonwetting.

(a) For complete wetting, the interfacial tensions follow Antonow's rule $\sigma_{\alpha \gamma}=\sigma_{\alpha \beta}+\sigma_{\beta \gamma}$. The $\beta$ phase spreads across the interface to form an intruding film separating the other two phases, as shown in Figure 1a.

(b) For partial wetting, the interfacial tensions obey the Neumann inequality ${ }^{3} \sigma_{\alpha \beta}-\sigma_{\beta \gamma}<\sigma_{\alpha \gamma}<\sigma_{\alpha \beta}+\sigma_{\beta \gamma}$. The $\beta$ phase forms a droplet suspended at the $\alpha-\gamma$ interface with a finite contact angle, as shown in Figure 1b.

(c) For nonwetting, the interfacial tensions follow Antonow's rule $^{2}$ either $\sigma_{\beta \gamma}=\sigma_{\alpha \beta}+\sigma_{\alpha \gamma}$ or $\sigma_{\alpha \beta}=\sigma_{\beta \gamma}+\sigma_{\alpha \gamma}$. A very small amount of $\beta$ phase would form a nonwetting droplet suspended at the $\alpha-\gamma$ interface, as shown in panels $\mathrm{c}$ and $\mathrm{d}$ of Figure 1. On the other hand, if the system is introduced to a substantial amount of $\beta$ phase, the lower $\alpha$ (or upper $\gamma$ ) phase would overcome gravity to form a thin intruding film separating the other phases $\beta$ and $\gamma$ (or $\alpha$ ). In terms of the $\alpha$ phase, the lower $\alpha$ phase exhibits a complete wetting behavior at the $\beta-\gamma$ interface as shown in Figure $1 \mathrm{c}^{\prime}$, when $\sigma_{\beta \gamma}=\sigma_{\alpha \beta}+\sigma_{\alpha \gamma}$. While $\sigma_{\alpha \beta}=\sigma_{\beta \gamma}+\sigma_{\alpha \gamma}$, a complete wetting $\gamma$ phase is observed at the $\alpha-\beta$ interface, as shown in Figure $1 d^{\prime}$.

For certain systems the $\beta$ phase can exhibit partial wetting, complete wetting, or nonwetting behavior at the $\alpha-\gamma$ interface under different thermodynamic conditions. The transition of the $\beta$ phase between different wetting behaviors is called a wetting

\footnotetext{
* Corresponding author. E-mail address: ljchen@ccms.ntu.edu.tw.
}

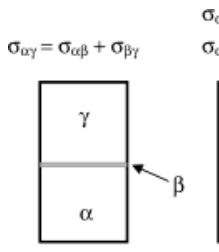

(a)

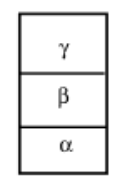

(a') $\sigma_{\alpha \gamma}<\sigma_{\alpha \beta}+\sigma_{\beta \gamma}$

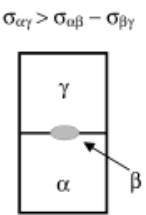

(b)

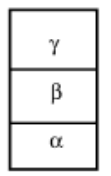

(b')

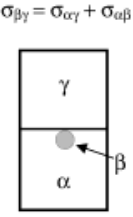

(c)

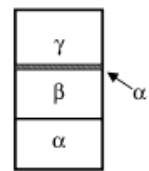

(c')

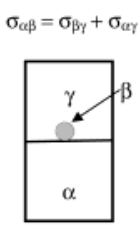

(d)

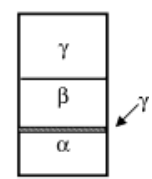

(d')
Figure 1. Wetting behavior at a fluid-fluid interface: (a) a complete wetting $\beta$ phase at the $\alpha-\gamma$ interface, (b) a partial wetting $\beta$ phase at the $\alpha-\gamma$ interface, (c) a nonwetting $\beta$ phase at the $\alpha-\gamma$ interface, (d) a nonwetting $\beta$ phase at the $\alpha-\gamma$ interface, ( $\left.\mathrm{c}^{\prime}\right)$ a complete wetting $\alpha$ phase at the $\beta-\gamma$ interface, and ( $\left.\mathrm{d}^{\prime}\right)$ a complete wetting $\gamma$ phase at the $\alpha-\beta$ interface. The expected condition for only a very small amount of the $\beta$ phase at the $\alpha \gamma$ interface is shown in the upper row, and the condition for a substantial amount of the $\beta$ phase at the $\alpha \gamma$ interface is shown in the lower row. The thickness of the $\alpha$ and $\gamma$ phase wetting layer in panels $\mathrm{c}^{\prime}$ and $\mathrm{d}^{\prime}$, respectively, is exaggerated for illustration.

transition. This remarkable interfacial phenomenon is the topic of extensive research both theoretically and experimentally due to its importance in many industrial applications. ${ }^{4}$

Particularly, the wetting behavior of water + oil $+\mathrm{C}_{i} \mathrm{E}_{j}$ mixtures has been extensively studied. Aratono and Kahlweit ${ }^{5}$ have reported that in the water + octane $+\mathrm{C}_{5} \mathrm{E}_{2}$ system, the middle $\mathrm{C}_{5} \mathrm{E}_{2}$-rich phase would completely wet the oil-water interface while the system approached either the upper (UCEP) or lower (LCEP) critical end points. That is, the behavior of the middle phase evolves from complete wetting to partial wetting and then to complete wetting along with increasing 
temperature. On the other hand, by measuring interfacial tensions and contact angles, Chen and $\mathrm{Yan}^{6}$ found that the middle $\mathrm{C}_{6} \mathrm{E}_{2}$-rich phase undergoes interfacial phase transitions: nonwetting $\rightarrow$ partial wetting $\rightarrow$ complete wetting at the oil-water interface in the three-liquid-phase coexisting region of the water + tetradecane $+\mathrm{C}_{6} \mathrm{E}_{2}$ system by simply tuning the system temperature. Note that the nonwetting behavior in the water + tetradecane $+\mathrm{C}_{6} \mathrm{E}_{2}$ system belongs to the type shown in Figure 1c. In addition, the observations of the wetting transitions of ternary water + oil $+\mathrm{C}_{i} \mathrm{E}_{j}$ systems have been extensively explored as the system is driven to either one of its critical end points by varying the strength of amphiphiles, ${ }^{5,7}$ by tuning the salt concentrations, ${ }^{8,9}$ by varying the temperature,,$^{5,6,10,11}$ and by varying the chain length of oils. ${ }^{12,13}$

Numerous theoretical approaches were proposed to describe the phase and interfacial behavior of ternary water + oil + amphiphile systems. By employing a simple lattice model, Schick and Shih ${ }^{14}$ studied a symmetrical case in which water and oil were interchangeable. It was shown that if the system is sufficiently close to the tricritical point, the amphiphile-rich phase wets the water-oil interface. However, when the system is far from the tricritical point, a complete wetting oil-rich phase or a complete wetting water-rich phase may also be observed. More sophisticated lattice models have recently been reviewed in detail by Gompper and Schick. ${ }^{15}$ Talanquer and Oxtoby ${ }^{16}$ have recently developed a simple off-lattice density functional theory and applied it to water + oil + amphiphile mixtures. Their model successfully resembles the phase behavior of ternary amphiphilic systems. For the chosen parameter set, these authors also found that the middle amphiphile-rich phase exhibits partial wetting at the oil-water interface over the whole three-liquid-phase coexisting region. ${ }^{16}$

In our previous study, ${ }^{17}$ we had applied a density functional theory to study wetting behaviors of binary associating mixtures which are described by the SAFT. In fact, the binary mixture water + amphiphile $\mathrm{C}_{i} \mathrm{E}_{j}$ is a typical example for binary associating mixtures. In this study, the model was further extended to explore the phase and wetting behaviors of ternary water + oil + amphiphile mixtures.

This paper is organized as follows. The Helmholtz free energy model is given in the next section. The results for the phase and wetting behaviors of ternary water + oil + amphiphile mixtures and comparisons with experimental results are presented in Section III. Finally in Section IV, we make some concluding remarks.

\section{Helmholtz Free Energy Model}

The SAFT was employed to describe the ternary mixture that is composed of equal-sized hard-sphere molecules $\mathrm{A}, \mathrm{B}$, and $\mathrm{C}$, representing water, amphiphile, and oil, respectively. The hydrogen bonding between water and amphiphile molecules was described by Wertheim's theory for associating fluids. Here we assume that each A and B molecule has only one active site that allows association (hydrogen bonding) between unlike pair molecules.

The SAFT bulk free energy is composed of the contributions of repulsive, attractive, and associating parts. The total Helmholtz free energy density of the mixture $f$ can be written as ${ }^{18}$

$$
f=f_{\mathrm{R}}+f_{\mathrm{M}}+f_{\mathrm{A}}
$$

where $f_{\mathrm{R}}$ is the repulsive contribution of the hard-sphere reference fluid, $f_{\mathrm{M}}$ is the van der Waals mean-field term due to the isotropic long-range attraction forces, and $f_{\mathrm{A}}$ is the contribution of the short-range association between molecules $\mathrm{A}$ and B.

The repulsive contribution is described by the CarnahanStarling expression ${ }^{19}$ for equal-sized hard-sphere mixtures with a diameter $d$

$$
f_{\mathrm{R}}=k T \sum_{i} \rho_{i}\left[\ln \rho_{i}-1+\frac{4 \eta-3 \eta^{2}}{(1-\eta)^{2}}\right] \quad(i=\mathrm{A}, \mathrm{B}, \mathrm{C})
$$

where $k$ is the Boltzmann constant, $T$ is the absolute temperature, $\rho_{i}$ is the number density of component $i$, and the packing fraction $\eta=\left(\pi d^{3} / 6\right) \sum_{i} \rho_{i}$.

The long-range attractive contribution is treated at the meanfield level ${ }^{20}$

$$
f_{\mathrm{M}}=-1 / 2 \sum_{i} \alpha_{i j} \rho_{i} \rho_{j}
$$

where the total strength $\alpha_{i j}$ is defined by

$$
\alpha_{i j}=-\int \mathrm{d} \mathbf{r} \Phi_{i j}(r) \quad(i, j=\mathrm{A}, \mathrm{B}, \mathrm{C})
$$

An isotropic interaction potential of inverse sixth power law decay is employed ${ }^{21}$

$$
\Phi_{i j}(r)=-4 \epsilon_{i j}\left(\frac{d}{r+v_{i j} d}\right)^{6} H(r-d)
$$

where $\epsilon_{i j}$ is the energy parameter, $H$ is the Heaviside step function, and $v_{i j}$ is the parameter used to adjust the range of attractive interaction between molecules $i$ and $j$. In this study, the total strength $\alpha_{i j}$ is fixed, hence the phase diagram, coexisting densities, and the critical end points remain the same as $v_{i j}$ varies. Any changes in the calculated interfacial properties must arise from changes in $v_{i j}$ of the potential, which does not alter the bulk phase equilibria. For a fixed $\alpha_{i j}$, the energy parameter $\epsilon_{i j}$ varies accordingly to the parameter $v_{i j}$. Note that a positive $v_{i j}$ would weaken the potential $\Phi_{i j}$ at short distances and enhance the potential $\Phi_{i j}$ at long distances. On the other hand, a negative $v_{i j}$ would enhance the potential $\Phi_{i j}$ at short distances and weaken the potential $\Phi_{i j}$ at long distances.

The association contribution is evaluated directly from Wertheim's first-order thermodynamic perturbation theory. For each A and B molecule with only one attractive bonding site, the association contribution to the free energy can be written as $^{18}$

$$
f_{\mathrm{A}}=k T \sum_{i} \rho_{i}\left[\ln \chi_{i}-\frac{\chi_{i}}{2}+\frac{1}{2}\right] \quad(i=\mathrm{A} \text { and } \mathrm{B})
$$

where $\chi_{i}$ is the fraction of nonbonded molecules of type $i$. These quantities are obtained by solving the following mass action equations simultaneously.

$$
\begin{aligned}
& \chi_{\mathrm{A}}=\frac{1}{1+\rho_{\mathrm{B}} \Delta_{\mathrm{AB}} \chi_{\mathrm{B}}} \\
& \chi_{\mathrm{B}}=\frac{1}{1+\rho_{\mathrm{A}} \Delta_{\mathrm{AB}} \chi_{\mathrm{A}}}
\end{aligned}
$$

The quantity $\Delta_{\mathrm{AB}}$ is approximated by $\Delta_{\mathrm{AB}}=4 \pi g^{\mathrm{HS}}(\mathrm{d}) K_{\mathrm{AB}^{-}}$ $\left[\exp \left(\epsilon_{\mathrm{W}} / k T\right)-1\right] .^{22}$ The symbol $K_{\mathrm{AB}}$ is the bonding volume and $\epsilon_{\mathrm{W}}$ is the energy parameter of association. In addition, $g^{\mathrm{HS}}(\mathrm{d})$ stands for the contact value of the radial distribution 


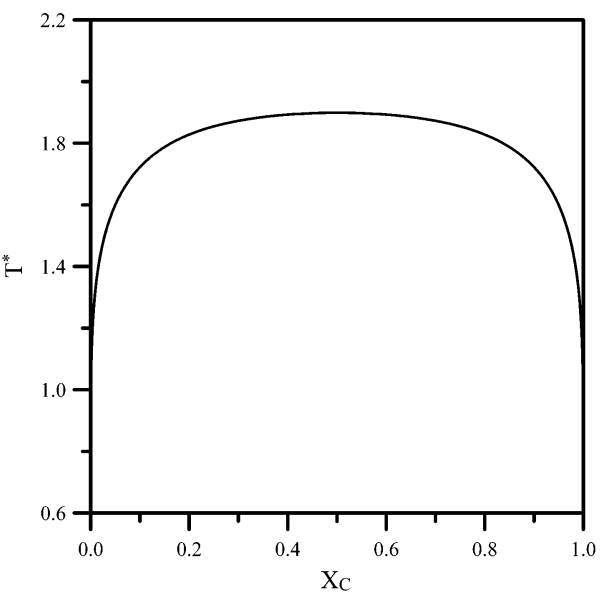

(a)

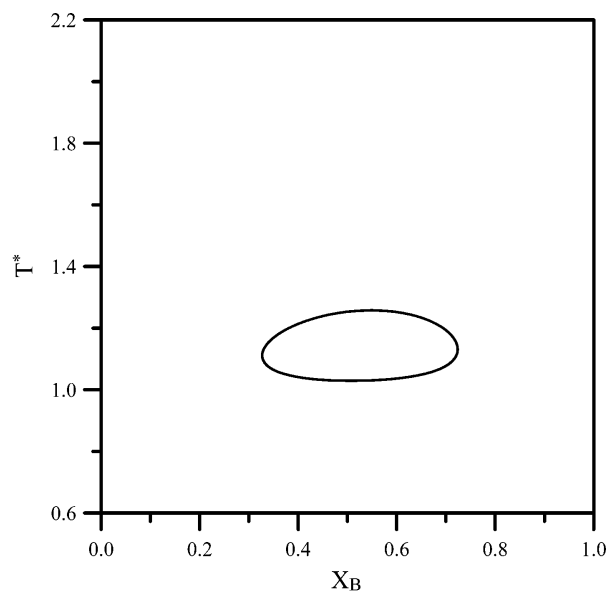

(b)

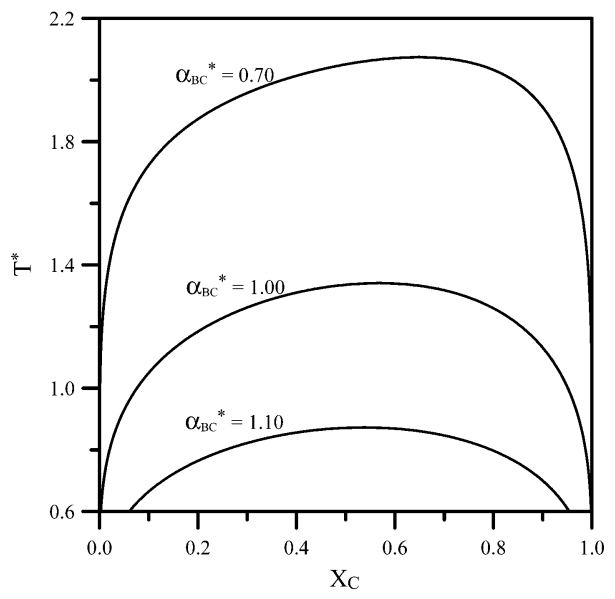

(c)

Figure 2. Phase diagrams of three binary mixtures: (a) $\mathrm{A}+\mathrm{C}$ mixture, (b) $\mathrm{A}+\mathrm{B}$ mixture, and (c) $\mathrm{B}+\mathrm{C}$ mixture with various $\alpha_{\mathrm{BC}}{ }^{*}$ at $P^{*}=$ 1.0.

function of the hard sphere fluid and is given by $g^{\mathrm{HS}}(\mathrm{d})=(1-$ $0.5 \eta) /(1-\eta)^{3} \cdot{ }^{19,23}$

\section{Results and Discussions}

All the calculations were performed in reduced units: $T^{*}=$ $k T / \epsilon_{\mathrm{AA}}, \mu_{i}^{*}=\mu_{i} / \epsilon_{\mathrm{AA}}, P^{*}=P d^{3} / \epsilon_{\mathrm{AA}}, \epsilon_{i j}^{*}=\epsilon_{i j} / \epsilon_{\mathrm{AA}}, \epsilon_{\mathrm{W}}^{*}=\epsilon_{\mathrm{W}} /$ $\epsilon_{\mathrm{AA}}, \alpha_{i j} *=3 \alpha_{i j} / 16 \pi d^{3} \epsilon_{\mathrm{AA}}, K_{\mathrm{AB}} *=K_{\mathrm{AB}} / d^{3}$, and $\rho_{i} *=\rho_{i} d^{3}$. Note that $K_{\mathrm{AB}} *=10^{-5}$ and $v_{\mathrm{AA}}=v_{\mathrm{BB}}=v_{\mathrm{CC}}=0$ in all our calculations. All the phase and wetting behaviors were examined at a constant pressure, $P^{*}=1.0$.

A. Phase Behavior. At a fixed temperature, the criteria for a multiphase equilibrium require that the chemical potential of each component and the pressure should be the same in all phases. The equations used to determine the bulk densities of the three-phase equilibrium system are given as follows

$$
\begin{gathered}
\mu_{i}\left(\rho_{\mathrm{A}}{ }^{\mathrm{I}}, \rho_{\mathrm{B}}{ }^{\mathrm{I}}, \rho_{\mathrm{C}}{ }_{\mathrm{I}}^{\mathrm{I}}, T\right)=\mu_{i}\left(\rho_{\mathrm{A}}{ }^{\mathrm{II}}, \rho_{\mathrm{B}}{ }^{\mathrm{II}}, \rho_{\mathrm{C}}{ }^{\mathrm{II}}, T\right)=\mu_{i}\left(\rho_{\mathrm{A}}{ }^{\mathrm{III}}, \rho_{\mathrm{B}}{ }^{\mathrm{III}}, \rho_{\mathrm{C}}{ }^{\mathrm{III}}, T\right) \\
P\left(\rho_{\mathrm{A}}{ }^{\mathrm{I}}, \rho_{\mathrm{B}}{ }^{\mathrm{I}}, \rho_{\mathrm{C}}{ }^{\mathrm{I}}, T\right)=P\left(\rho_{\mathrm{A}}{ }^{\mathrm{II}}, \rho_{\mathrm{B}}{ }^{\mathrm{II}}, \rho_{\mathrm{C}}{ }^{\mathrm{II}}, T\right)= \\
P\left(\rho_{\mathrm{A}}{ }^{\mathrm{III}}, \rho_{\mathrm{B}}{ }^{\mathrm{III}}, \rho_{\mathrm{C}}{ }^{\mathrm{III}}, T\right)=P_{\text {given }}
\end{gathered}
$$

where the superscripts I, II, and III stand for three coexisting phases.
The chemical potential of component $i$ is given by

$$
\mu_{i}=\left(\frac{\partial f}{\partial \rho_{i}}\right)_{T, V, \rho_{j \neq i}}
$$

and the equilibrium pressure is thus obtained by

$$
P=\sum_{i} \mu_{i} \rho_{i}-f
$$

Before examining the phase behavior of ternary A + B + C mixtures, we started with studying the phase diagrams of the three binary mixtures: $\mathrm{A}+\mathrm{C}, \mathrm{A}+\mathrm{B}$, and $\mathrm{B}+\mathrm{C}$. Note that molecules $\mathrm{A}, \mathrm{B}$, and $\mathrm{C}$ represent water, amphiphile, and oil, respectively.

(i) For the binary $\mathrm{A}+\mathrm{C}$ mixture, the parameters $\alpha_{\mathrm{AA}} *=$ $\alpha_{\mathrm{CC}} *=1.0$ and $\alpha_{\mathrm{AC}} *=0.45$ are chosen to indicate the very low mutual solubility between water and oil. The upper critical solution temperature (UCST) of this mixture occurs at $T^{*}=$ 1.899, as shown in Figure 2a.

(ii) For the binary $\mathrm{A}+\mathrm{B}$ mixture, the parameters $\alpha_{\mathrm{BB}} *=$ 1.4 and $\alpha_{\mathrm{AB}} *=1.0$ are selected to represent the relatively high mutual solubility between water and amphiphile. In addition, a rather strong associating effect, $\epsilon_{\mathrm{W}} *=8.25$, is introduced to accomplish a closed-loop phase diagram that is bounded by a 
$\underline{2} \phi$

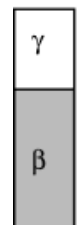

$3 \phi$

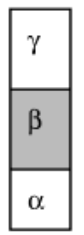

$\overline{2} \phi$

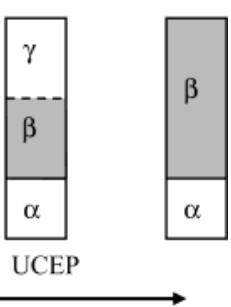

Increasing temperature
Figure 3. Variation of the number of phases as a function of temperature.

UCST at $T^{*}=1.258$ and a lower critical solution temperature (LCST) at $T^{*}=1.029$, as illustrated in Figure 2b.

(iii) For the binary $\mathrm{B}+\mathrm{C}$ mixture, the parameters $\alpha_{\mathrm{BB}} *=$ 1.4 and $\alpha_{\mathrm{CC}} *=1.0$, and the attraction energy parameter $\alpha_{\mathrm{BC}} *$ is considered as an adjustable parameter in this study. Figure $2 \mathrm{c}$ illustrates the phase diagram of $\mathrm{B}+\mathrm{C}$ mixture with various $\alpha_{\mathrm{BC}} *$. The immiscibility gap shrinks dramatically as $\alpha_{\mathrm{BC}} *$ increases from 0.70 to 1.10 .

All the phase behaviors of the three binary mixtures are qualitatively consistent with the experimental observation of water + oil $+\mathrm{C}_{i} \mathrm{E}_{j}$ mixtures. ${ }^{24}$

It is well understood that the phase behavior of the ternary system water + oil + amphiphile depends on temperature, pressure, and the nature of the components. ${ }^{24}$ The various phase equilibria of such systems can be grouped into four types, according to Winsor's classification. ${ }^{25}$

(i) Winsor type I phase equilibrium consists of two phases, an aqueous phase containing most of the amphiphile and an oil-rich phase.

(ii) Winsor type II phase equilibrium also consists of two phases, an oil-rich phase containing most of the amphiphile and an aqueous phase.

(iii) Winsor type III phase equilibrium consists of three phases, an aqueous phase and an oil-rich phase in coexistence with an amphiphile-rich phase containing appreciable amounts of oil and water.

(iv) Winsor type IV phase equilibrium has only a single homogeneous phase in which water, oil, and amphiphile are mutually solubilized.

At constant pressures, these four types of phase equilibria can be observed in water + oil $+\mathrm{C}_{i} \mathrm{E}_{j}$ systems by simply varying the temperature. By simply varying the temperature at a certain fixed concentration, one finds Winsor type I phase $(\overline{2} \phi)$ equilibria at low temperatures, Winsor type II phase $(\overline{2} \phi)$ equilibria at high temperatures, and Winsor type III phase $(3 \phi)$ equilibria at intermediate temperatures. Figure 3 schematically shows the variation of the number of phases as a function of temperature. Note that the three phases $\alpha, \beta$, and $\gamma$ denote respectively the water-rich, amphiphile-rich, and oil-rich phase. It is interesting to note that the amphiphile transfers continuously from the aqueous phase to the oil-rich phase as the temperature rises, i.e., the mutual solubility between amphiphile and oil increases with rising temperature. In other words, the amphiphile hydrophobicity increases along with temperature. Winsor type IV phase $(1 \phi)$ equilibria usually exist at the region of high amphiphile concentrations. The highest and the lowest temperatures of the three-liquid-phase coexisting body $(3 \phi)$ correspond to the upper (UCEP) and lower (LCEP) critical end points. In our calculation, both UCEP and LCEP are evaluated to

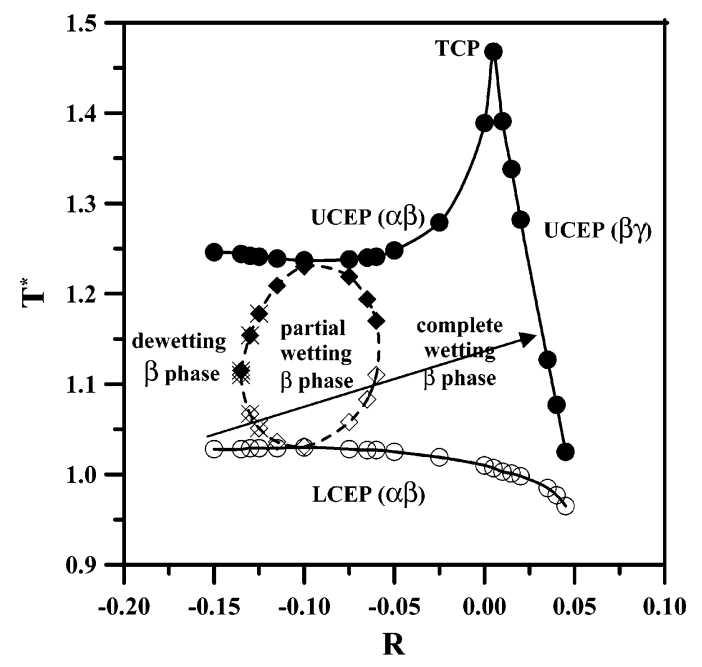

Figure 4. The effect of the amphiphile hydrophobicity $R$ on the critical end points and the wetting transition temperatures: UCEP $(\bullet)$, LCEP $(\bigcirc)$, upper wetting transition temperature $(\square)$, and lower wetting transition temperature $(\square)$. All the wetting transitions are second order, except those indicated by the symbol $\mathrm{X}$, which are first-order wetting transitions.

illustrate the temperature range of the three-liquid-phase coexisting body.

In our model, a parameter $R$ is introduced to represent the amphiphile hydrophobicity and defined as the following.

$$
\frac{R=}{\frac{\left.2\left(2 \alpha_{\mathrm{BC}} *-\alpha_{\mathrm{CC}} *-\alpha_{\mathrm{BB}} *\right)-\left(2 \alpha_{\mathrm{AB}} *-\alpha_{\mathrm{AA}} *-\alpha_{\mathrm{CB}} *\right)\right]}{4}-2 \alpha_{\mathrm{AB}} *+\alpha_{\mathrm{AA}} *}(12)
$$

The parameter $R$ stands for the relative strength of the attractive interaction between water (A) and amphiphile (B) molecules to that of oil (C) and amphiphile (B) molecules. When the parameter $R$ is increased, the amphiphile becomes more hydrophobic. Consequently, the mutual solubility between amphiphile and oil is increased. On the other hand, when the parameter $R$ is decreased, the amphiphile becomes more hydrophilic and the mutual solubility between amphiphile and water is enhanced.

Figure 4 shows the calculation results of both the critical end points, UCEP and LCEP, i.e., the temperature range of the threeliquid-phase coexisting body of the ternary A $+\mathrm{B}+\mathrm{C}$ mixture as a function of the amphiphile hydrophobicity $R$. When $R<$ -0.05 , the phase behavior is dominated by the binary $\mathrm{A}+\mathrm{B}$ mixture at a given value of $R$. That is, the occurrence of both the upper and the lower critical end points is simply due to the mergence of A-rich $(\alpha)$ and B-rich $(\beta)$ phases into a single phase. Since both the attractive interactions $\alpha_{\mathrm{AC}} *$ and $\alpha_{\mathrm{BC}} *$ are very weak in this region, the component $\mathrm{C}$ is almost like an inert substance to the binary $\mathrm{A}+\mathrm{B}$ mixture. As a consequence, the introduction of the component $\mathrm{C}$ to the binary $\mathrm{A}+\mathrm{B}$ system has little effect on the closed-loop phase diagram of the binary $\mathrm{A}+\mathrm{B}$ mixture, as shown in Figure $2 \mathrm{~b}$. The variation of UCEPs and LCEPs as a function of $R$ is quite small.

With increasing $R$, the component $\mathrm{B}$ becomes more hydrophobic and the attractive interaction between molecules $\mathrm{C}$ and $\mathrm{B}$ becomes comparable to that of molecules $\mathrm{A}$ and $\mathrm{B}$. The critical phases of the UCEP would evolve from $\alpha / \beta$ to $\beta / \gamma$ along with further increasing $R$. These two $(\alpha / \beta$ and $\beta / \gamma)$ critical end 


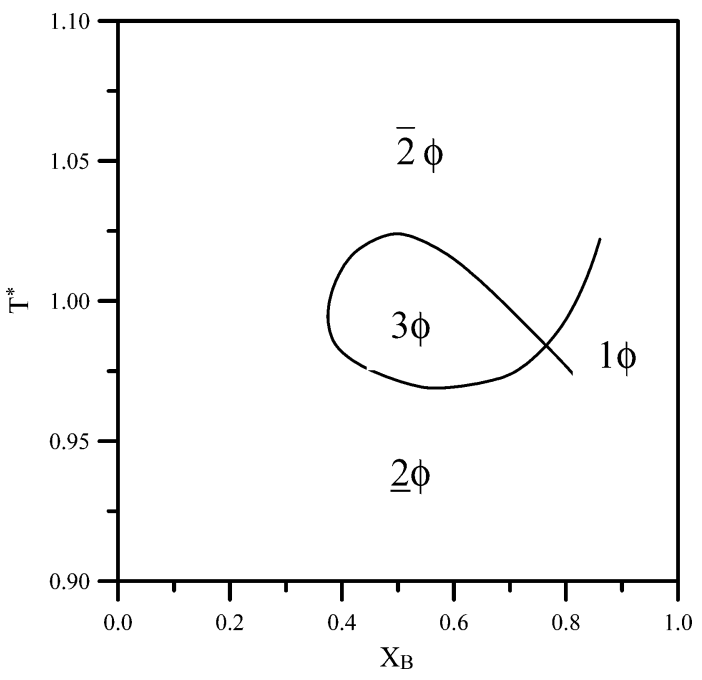

Figure 5. The fish-shaped phase diagram at $R=0.045$.

points merge into a tricritical point. In addition, the LCEP is also no longer predominated only by the $\mathrm{A}-\mathrm{B}$ association and decreases with increasing $R$. A further increase in $R$ would shrink the three-phase coexisting temperature region. A conventional fish-shaped phase diagram can be observed for $R>$ 0.01 . Figure 5 shows the fish-shaped phase diagram for $R=$ 0.045 , consistent with the experimental observation in water + oil $+\mathrm{C}_{i} \mathrm{E}_{j}$ mixtures. ${ }^{24}$ Also at a fixed concentration, e.g. $X_{\mathrm{B}}=$ 0.6 in Figure 5, we are able to find the evolution of phase behavior, $2 \phi \rightarrow 3 \phi \rightarrow \overline{2} \phi$, along with increasing temperature, consistent with the phase behavior described in Figure $3 .^{24}$

Note that Talanquer and Oxtoby ${ }^{16}$ also applied the SAFT to successfully mimic the phase behavior of ternary amphiphilic systems. Essentially, the SAFT employed in this study is almost exactly the same as that of Talanquer and Oxtoby, ${ }^{16}$ except for the screening parameter $\delta_{\mathrm{AC}}$. Talanquer and Oxtoby ${ }^{16}$ introduced the screening parameter $\delta_{\mathrm{AC}}$ to vary the screening effect of interactions between water and oil due to the presence of amphiphile. However, the screening parameter $\delta_{\mathrm{AC}}$ is turned off in this study for simplicity. On the other hand, the parameter $v_{i j}$ is introduced in eq 5 in this study, and is always equal to zero in the model of Talanquer and Oxtoby. ${ }^{16}$ In addition, Talanquer and Oxtoby ${ }^{16}$ selected the parameters $\epsilon_{\mathrm{BB}} *=2.0$ and $\epsilon_{\mathrm{AB}} *=1.35$, which are quite different from the ones used in this study $\left(\epsilon_{\mathrm{BB}} *=1.4\right.$ and $\left.\epsilon_{\mathrm{AB}} *=1.0\right)$. Essentially, a relatively weaker surfactant is used in this study than in that of Talanquer and Oxtoby. ${ }^{16}$ As one can see, the closed-loop in Figure $2 \mathrm{~b}$ is smaller that of Figure $1 \mathrm{~b}$ in ref 16.

As mentioned above, the amphiphile hydrophobicity increases along with raising temperature. Presumably, the variation of the amphiphile hydrophobicity $R$ as a function of temperature is schematically followed as the arrow shows in Figure 4. Note that the evolution of phase behavior: $2 \phi \rightarrow 3 \phi \rightarrow \overline{2} \phi$ along with increasing temperature is also successfully predicted with this temperature-dependent parameter $R$, consistent with the phase behavior of water + oil + amphiphile mixtures. ${ }^{24} \mathrm{~A}$ fishshaped phase diagram is also obtained while the temperaturedependent parameter $R$ is considered.

B. Wetting Behavior. The properties of liquid-liquid interfaces between coexisting phases of ternary mixtures can be calculated with the application of density functional theory. ${ }^{26}$ The total Helmholtz free energy density of an inhomogeneous ternary mixture can be expressed as a function of the local densities $\rho_{i}(\mathbf{r})$

$$
\begin{aligned}
& F\left(\rho_{i}(\mathbf{r})\right)=\int_{V} \mathrm{~d} \mathbf{r} f_{\mathrm{R}}\left(\rho_{i}(\mathbf{r})\right)+\int_{V} \mathrm{~d} \mathbf{r} f_{\mathrm{A}}\left(\rho_{i}(\mathbf{r})\right)+ \\
& 1 / 2 \sum_{i j} \iint_{V} \mathrm{~d} \mathbf{r} \mathrm{d} \mathbf{r}^{\prime} \phi_{i j}\left(\left|\mathbf{r}-\mathbf{r}^{\prime}\right|\right) \rho_{i}(\mathbf{r}) \rho_{j}\left(\mathbf{r}^{\prime}\right)
\end{aligned}
$$

where $f_{\mathrm{R}}\left(\rho_{i}(\mathbf{r})\right)$ and $f_{\mathrm{A}}\left(\rho_{i}(\mathbf{r})\right)$ are considered to be functions of local densities $\rho_{i}(\mathbf{r})$ and are given by eqs 2 and 6 , respectively.

The grand potential functional $\Omega\left(\rho_{i}(\mathbf{r})\right)$ for an inhomogeneous ternary mixture can be written as follows

$$
\Omega\left(\rho_{i}(\mathbf{r})\right)=F\left(\rho_{i}(\mathbf{r})\right)-\sum_{i} \mu_{i} \int_{V} \mathrm{~d} \mathbf{r} \rho_{i}(\mathbf{r})
$$

where $V$ is the system volume. The equilibrium density profiles across a planar interface are obtained through the minimization of $\Omega\left(\rho_{i}(\mathbf{r})\right) .{ }^{26}$ The derivative of $\Omega\left(\rho_{i}(\mathbf{r})\right)$ with respect to $\rho_{i}(\mathbf{r})$ is equal to zero, yielding a set of integral equations at equilibrium chemical potentials $\mu_{i}$

$$
\begin{array}{r}
\mu_{\mathrm{R}}\left(\rho_{i}(\mathbf{r})\right)+\mu_{\mathrm{A}}\left(\rho_{i}(\mathbf{r})\right)=\mu_{i}-\sum_{i j} \int_{V} \mathrm{~d} \mathbf{r}^{\prime} \phi_{i j}\left(\left|\mathbf{r}-\mathbf{r}^{\prime}\right|\right) \rho_{j}\left(\mathbf{r}^{\prime}\right) \\
(i=\mathrm{A}, \mathrm{B}, \mathrm{C})
\end{array}
$$

where $\mu_{\mathrm{R}}\left(\rho_{i}(\mathbf{r})\right)=\partial f_{\mathrm{R}}\left(\rho_{i}(\mathbf{r})\right) / \partial \rho_{i}(\mathbf{r})$ and $\mu_{\mathrm{A}}\left(\rho_{i}(\mathbf{r})\right)=\partial f_{\mathrm{A}}\left(\rho_{i}(\mathbf{r})\right) /$ $\partial \rho_{i}(\mathbf{r})$.

The last equation can be solved numerically by an iterative method. ${ }^{21}$ The boundary conditions of the Euler-Lagrange equations, eq 15, are the equilibrium densities of the bulk phases, which are evaluated from the SAFT equation of state via the method mentioned in the previous section. Once the equilibrium density profiles are determined, the interfacial tension $\sigma$ is directly evaluated from

$$
\sigma=\frac{\Omega\left(\rho_{i}(\mathbf{r})\right)-P V}{A}
$$

where the symbol $A$ represents the surface area of the planar interface between coexisting phases. Once three interfacial tensions of the three-phase coexisting mixture are obtained, the wetting behavior can be determined by examining three interfacial tensions that obey either Neumann's inequality ${ }^{3}$ or Antonow's rule. ${ }^{2}$

The wetting behavior of the ternary $\mathrm{A}+\mathrm{B}+\mathrm{C}$ mixture is also illustrated in Figure 4. The three-phase region is divided into four regions according to the wetting behavior.

(i) When $R \leq-0.15$, three interfacial tensions always obey Antonow's rule, $\sigma_{\beta \gamma}=\sigma_{\alpha \gamma}+\sigma_{\beta \gamma}$. In this case, the lower $\alpha$ phase completely wets the $\beta-\gamma$ interface over the entire threephase coexisting region, as shown in Figure $1 c^{\prime}$. In other words, the middle $\beta$ phase exhibits a nonwetting behavior at the $\alpha-\gamma$ interface, as shown in Figure 1c.

(ii) When $-0.15<R<-0.10$, a wetting transition of the $\beta$ phase at the $\alpha-\gamma$ interface from partial to nonwetting behavior always occurs as the system is brought to either one of its critical end points. One can observe a sequence of wetting transitions of the $\beta$ phase, nonwetting $\rightarrow$ partial wetting $\rightarrow$ nonwetting, along with increasing temperature. Both the upper wetting temperature $\left(T_{\mathrm{WU}}\right)$ and lower wetting temperature $\left(T_{\mathrm{WL}}\right)$ approach their corresponding CEPs as the amphiphile hydrophobicity $R$ increases. Eventually, $T_{\mathrm{WU}}$ and $T_{\mathrm{WL}}$ would almost merge into UCEP and LCEP, respectively, at around $R=-0.10$. That is, the $\beta$ phase retains partial wetting behavior over the three- 
phase coexisting region at $R=-0.10$, except very close to its critical end points.

(iii) When $-0.10<R<-0.05$, a wetting transition of the $\beta$ phase at the $\alpha-\gamma$ interface from partial to complete wetting behavior always occurs as the system is brought to either one of its critical end points. Therefore, a sequential wetting transition, complete wetting $\rightarrow$ partial wetting $\rightarrow$ complete wetting, occurs with raising temperature. The regime of complete wetting $\beta$ phase enlarges and the temperature range of partial wetting $\beta$ phase shrinks as the amphiphile hydrophobicity $R$ increases.

(iv) When $R \geq-0.05$, three interfacial tensions always obey Antonow's rule, ${ }^{2} \sigma_{\alpha \gamma}=\sigma_{\alpha \beta}+\sigma_{\beta \gamma}$. That is, the $\beta$ phase always wets the $\alpha-\gamma$ interface over the whole three-phase coexisting region.

It is interesting to note that a simple off-lattice density functional theory of Talanquer and Oxtoby ${ }^{16}$ predicts that the middle amphiphile-rich phase exhibits partial wetting at the oilwater interface over the whole three-liquid-phase coexisting region of water + oil + amphiphile mixtures. That is very likely due to introducing a stronger amphiphile in their calculation, ${ }^{16}$ as mentioned above.

Note that the wetting behavior of the $\beta$ phase at the $\alpha-\gamma$ interface is strongly related to the amphiphile hydrophobicity $R$. When $R$ decreases, the amphiphile B becomes more hydrophilic and the $\beta$ phase tends to dewet the $\alpha-\gamma$ interface. On the other hand, as $R$ increases, the amphiphile $\mathrm{B}$ becomes more hydrophobic and the $\beta$ phase tends to completely wet the $\alpha-\gamma$ interface. As mentioned above, the amphiphile hydrophobicity increases as raising temperature. Presumably, the variation of the amphiphile hydrophobicity $R$ as a function of temperature is schematically followed as shown by the arrow in Figure 4. As a consequence, the wetting behavior of the $\beta$ phase at the $\alpha-\gamma$ interface would exhibit the following variations: nonwetting $\rightarrow$ partial wetting $\rightarrow$ complete wetting, along with increasing temperature. This prediction is consistent with the experimental observation in the three-liquid-phase coexisting region of the ternary water + alkane $+\mathrm{C}_{6} \mathrm{E}_{2}$ mixture..$^{10,13}$

Figures 6-8 illustrate the typical density profiles of three interfaces at $T^{*}=1.10$ for three different wetting behaviors of $\beta$ phase: nonwetting $(R=-0.15$, Figure 6$)$, partial wetting $(R$ $=-0.10$, Figure 7$)$, and complete wetting $(R=-0.05$, Figure $8)$. As one can see in Figure $6 c$, the shoulders in the density profiles are at the densities equivalent to the bulk $\alpha$ phase. The densities of the $\alpha$ phase are also shown in Figure 6, panels a and $\mathrm{b}$. That is, a well-defined thin film of $\alpha$ phase is developed at the $\beta-\gamma$ interface. On the other hand, the shoulders in the density profiles shown in Figure $8 \mathrm{~b}$ are at the densities equivalent to the bulk $\beta$ phase. That is, a well-defined thin film of the $\beta$ phase is developed at the $\alpha-\gamma$ interface. For the partial wetting $\beta$ phase, the shoulders in the density profiles across the $\alpha-\gamma($ or $\beta-\gamma)$ interface shown in Figure 7b (or Figure 7c) are at densities smaller than that of the bulk $\beta$ (or $\alpha$ ) phase.

In this study, the order of wetting transitions is also determined by strictly following the method of Tarazona and Evans. ${ }^{27} \mathrm{~A}$ wetting transition is said to be first order if the temperature dependence of wetting film thickness exhibits a discontinuity at the wetting transition temperature. On the other hand, if the wetting film thickness grows gradually and diverges at the wetting transition temperature, the wetting transition is identified as second order.

It was found ${ }^{17,21}$ that the attractive range parameter $v_{i j}$, defined in eq 5, has a strong effect on both the wetting transition temperature and the order of wetting transitions. In this study, (a)

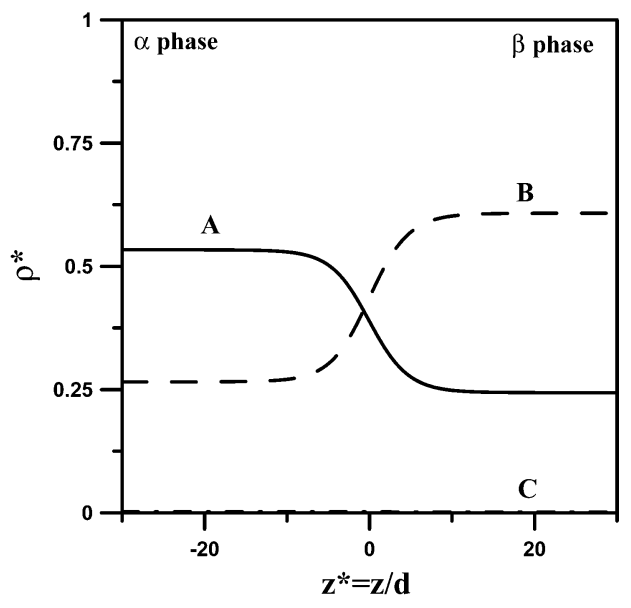

(b)

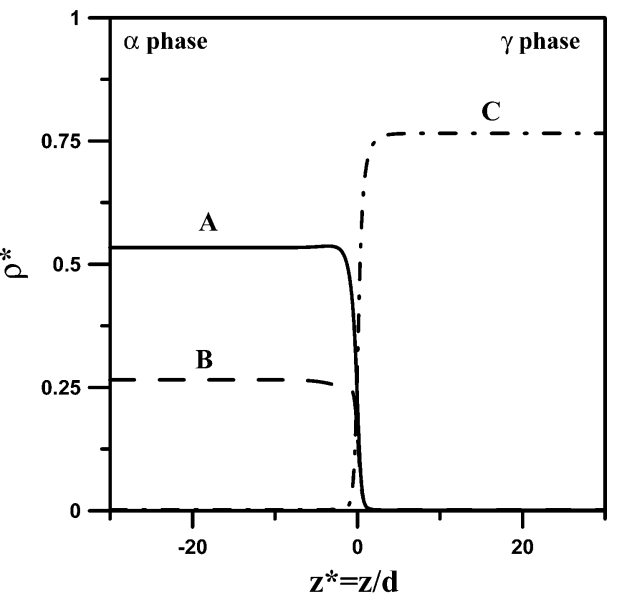

(c)

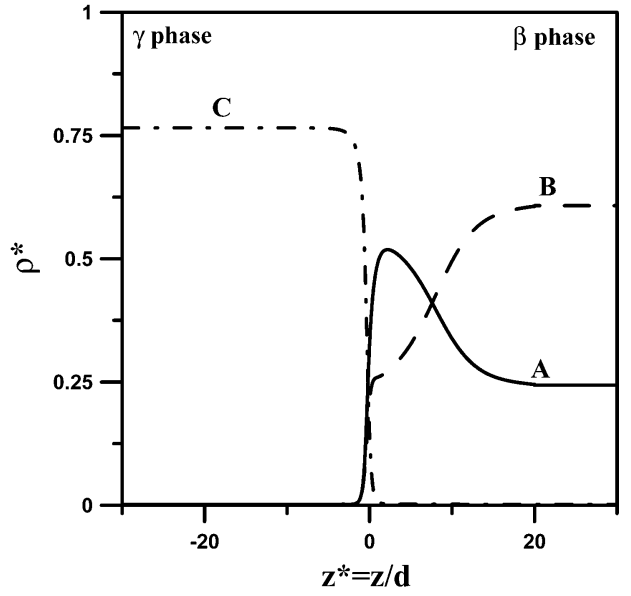

Figure 6. Density profiles of the (a) $\alpha-\beta$, (b) $\alpha-\gamma$, and (c) $\beta-\gamma$ interfaces at $T^{*}=1.10$ and $R=-0.15$. The $\beta$ phase exhibits nonwetting behavior at the $\alpha-\gamma$ interface. In other words, the $\alpha$ phase exhibits complete wetting behavior at the $\beta-\gamma$ interface, as illustrated in Figure $1 c^{\prime}$.

this effect of $v_{i j}$ was carefully examined for two cases, $R=$ -0.125 and -0.075 , which fall into the wetting behavior regions, respectively, (ii) and (iii) mentioned above.

Figure 9a illustrates our calculation results for various $v_{\mathrm{AB}}$ at $R=-0.125$. As one can see in Figure 9a, the increase in $v_{\mathrm{AB}}$ would narrow down the temperature window of the partial wetting $\beta$ phase and the wetting transition remains first order over the range of $v_{\mathrm{AB}}$. When $v_{\mathrm{AB}}$ is further increased beyond 0.04 , the middle $\beta$ phase does not wet the $\alpha-\gamma$ interface over the entire three-phase coexisting region. Figure $9 \mathrm{~b}$ illustrates the effect of $\nu_{\mathrm{BC}}$ on the wetting transition temperatures. The 
(a)

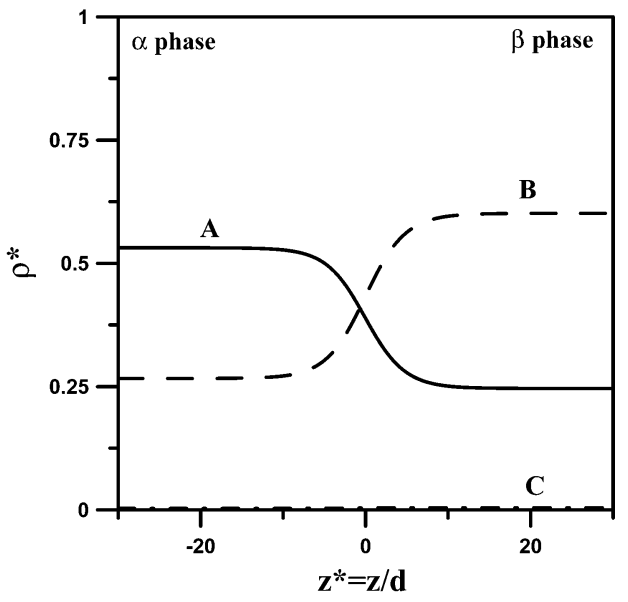

(b)

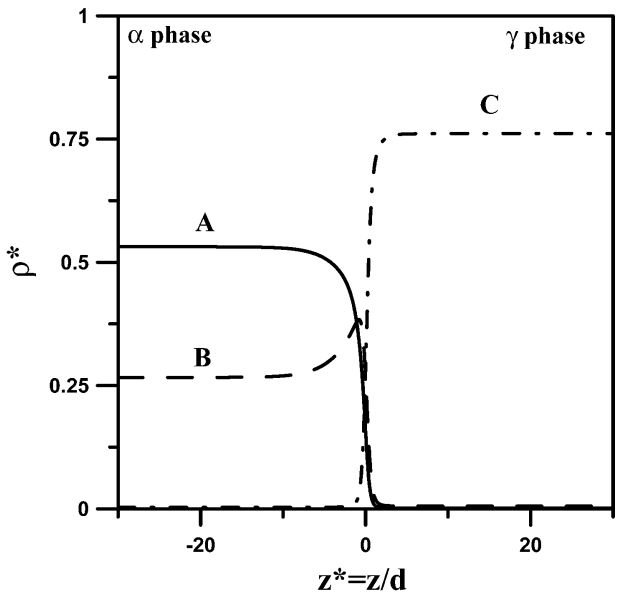

(c)

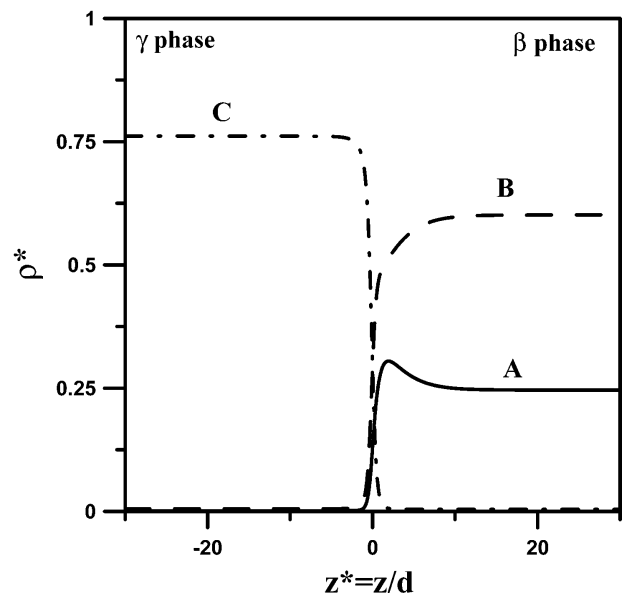

Figure 7. Density profiles of the (a) $\alpha-\beta$, (b) $\alpha-\gamma$, and (c) $\beta-\gamma$ interfaces at $T^{*}=1.10$ and $R=-0.10$. The $\beta$ phase exhibits partial wetting behavior at the $\alpha-\gamma$ interface.

temperature window of the partial wetting $\beta$ phase would enlarge along with increasing $v_{\mathrm{BC}}$. It is plausible that the formation of the $\alpha$ phase intruding layer at the $\beta-\gamma$ interface is inhibited by the enhancement of the attractive potential between molecules $\mathrm{B}$ and $\mathrm{C}$ at longer distances with increasing $\nu_{\mathrm{BC}}$. In addition, all the wetting transitions remain second order in all $\nu_{\mathrm{BC}}$ values.

Figure 10 illustrates the effects of $v_{\mathrm{AB}}$ and of $\nu_{\mathrm{BC}}$ on the wetting transition temperature and the order of wetting transitions at $R=-0.075$. The variations of the wetting transition temperature and of the order of transitions as a function of the attractive range parameter $v_{\mathrm{AB}}$ are very small, as shown in Figure 10a. Since the attractive strength $\alpha_{A C} *$ is small and both $\alpha_{A B}$ * and $\alpha_{\mathrm{BC}}{ }^{*}$ are relatively large, the $\beta$ phase is energetically (a)

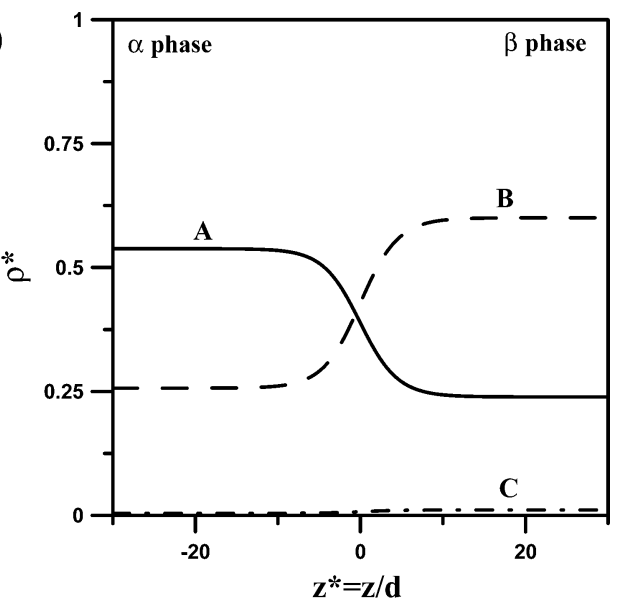

(b)

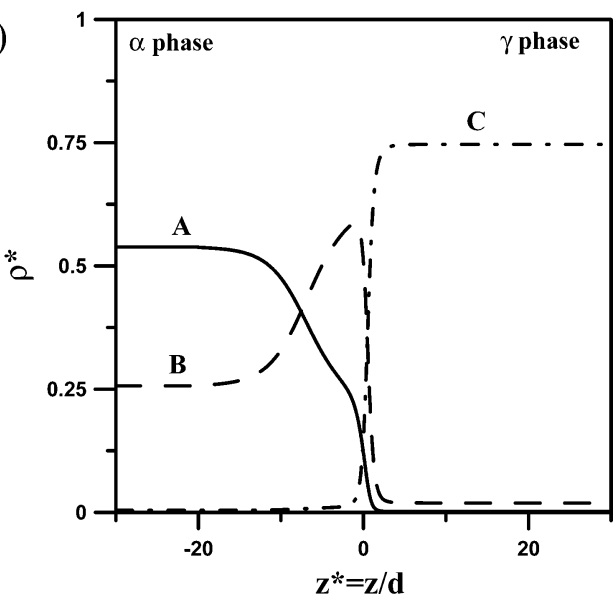

(c)

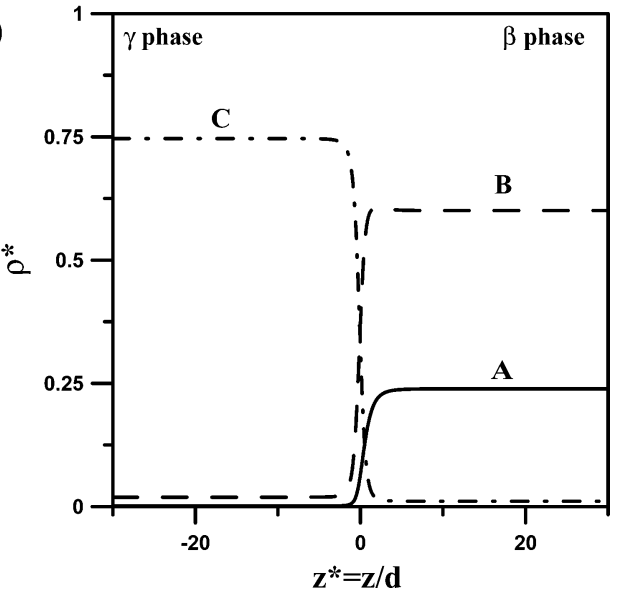

Figure 8. Density profiles of the (a) $\alpha-\beta$, (b) $\alpha-\gamma$, and (c) $\beta-\gamma$ interfaces at $T^{*}=1.10$ and $R=-0.05$. The $\beta$ phase exhibits complete wetting behavior at the $\alpha-\gamma$ interface.

favorable to intrude between the $\alpha-\gamma$ interface. Variation of the attractive range parameter $v_{\mathrm{AB}}$ only alters the range of interaction between $\mathrm{A}$ and $\mathrm{B}$ that has a very small effect on the wetting transition temperature. It should be noted that the wetting transition temperature is the outcome of the competition between the attractive strengths $\alpha_{A B} *$ and $\alpha_{B C} *$. In addition, both upper and lower wetting transitions remain second order for various $v_{\mathrm{AB}}$ values. Figure $10 \mathrm{~b}$ illustrates that the $\beta$ phase would favor the complete wetting behavior at the $\alpha-\gamma$ interface with an increase in $v_{\mathrm{BC}}$. This trend is similar to the case of $R=-0.125$ where the $\alpha$ phase would favor the complete wetting behavior at the $\beta-\gamma$ interface with an increase in $\nu_{\mathrm{AB}}$, as mentioned above. 
(a)
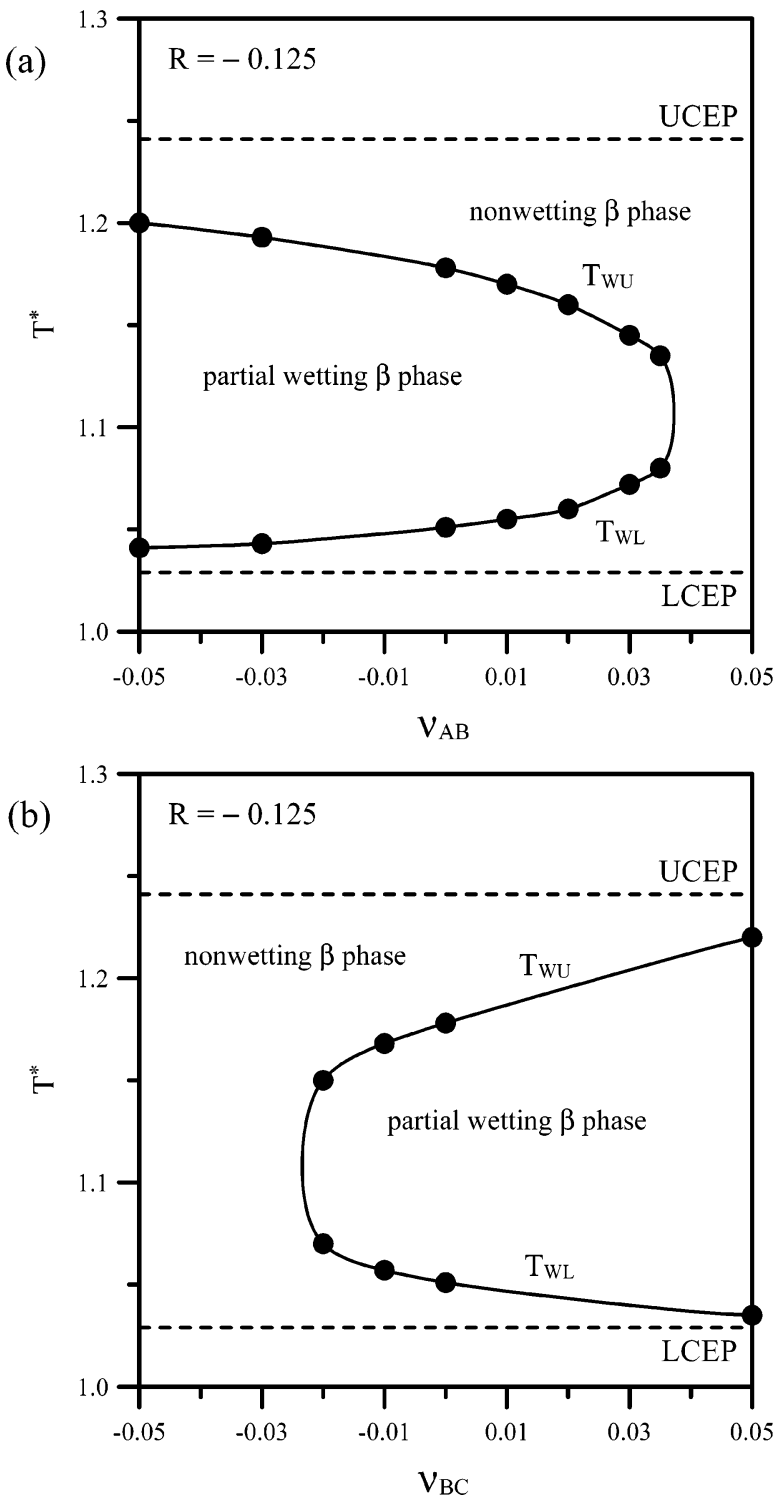

Figure 9. Variation of the wetting transition temperatures for $R=$ -0.125 as a function of (a) $\nu_{\mathrm{AB}}$ and (b) $\nu_{\mathrm{BC}}$ at $P^{*}=1.0$. The filled symbols represent first-order wetting transitions.

Note that all the wetting transitions in Figure 4 are calculated under the condition of $v_{i j}=0$. It is found that when $R>-0.125$, all the wetting transitions are second order and first order for $R$ $\leq-0.125$, as shown in Figure 4 .

The attractive interaction between molecules A and B consists of the van der Waals attraction and the short-ranged association interaction. It is found that the order of a wetting transition is the outcome of the competition between the attractive potential $\Phi_{i j}$ and the association interaction $\epsilon_{\mathrm{W}}{ }^{*} .{ }^{17,21}$ When the amphiphile hydrophobicity $R$ becomes smaller, the attractive interaction between molecules $\mathrm{A}$ and $\mathrm{B}$ is enhanced, and consequently would favor a first-order wetting transition. On the other hand, a large $\epsilon_{\mathrm{W}} *$ would introduce a strong short-ranged association between molecules $\mathrm{A}$ and $\mathrm{B}$ and consequently would favor a second-order wetting transition. At a fixed temperature, say $T^{*}$ $=1.1$, two wetting transitions occur as the parameter $R$ varies, as shown in Figure 4. As a result, the wetting transition is second order at larger $R$ and the other wetting transition switches to first order at smaller $R$, under the condition of a fixed association strength $\epsilon_{\mathrm{W}}{ }^{*}$. This finding is consistent with our previous study on the binary associating mixture. ${ }^{17}$
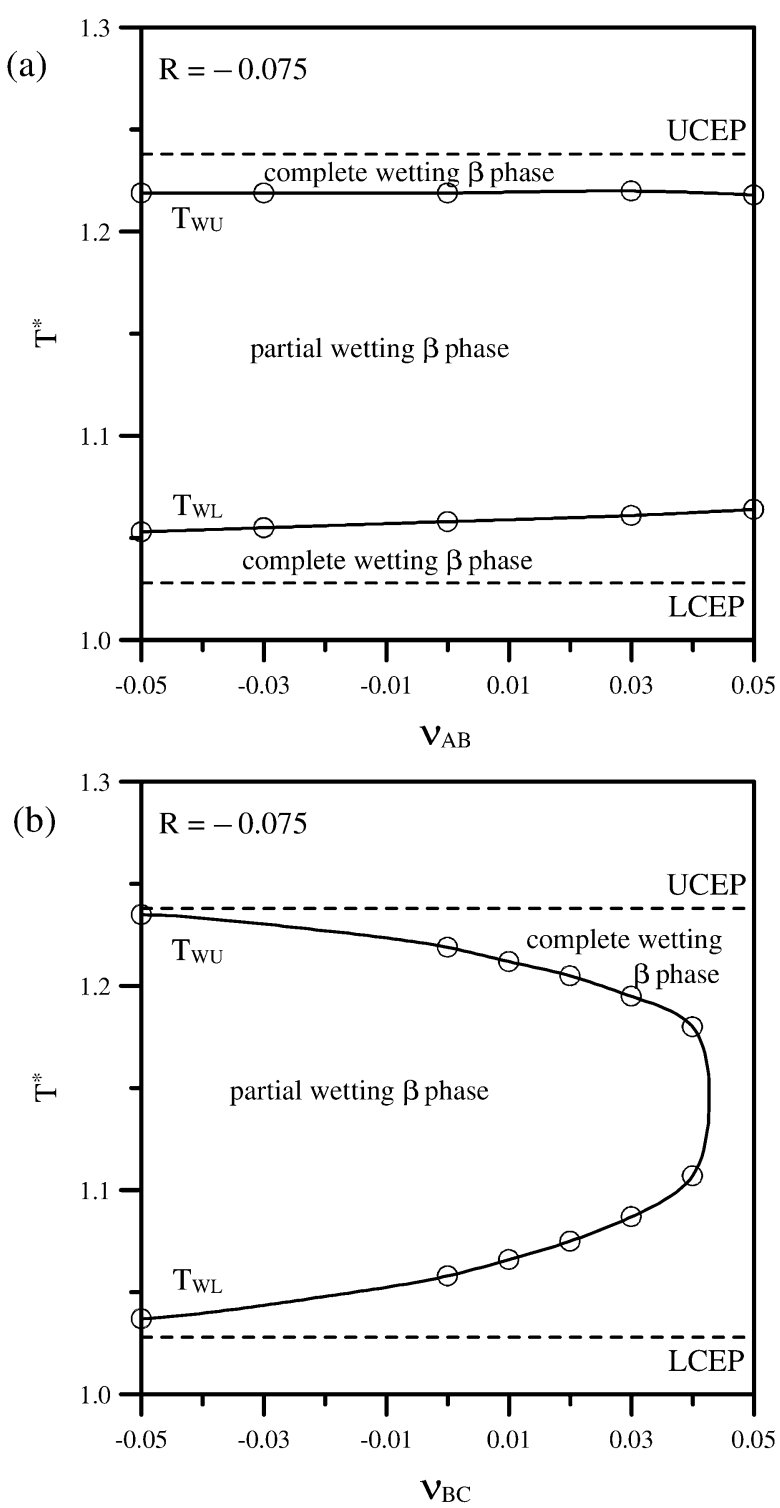

Figure 10. Variation of the wetting transition temperatures for $R=$ -0.075 as a function of (a) $\nu_{\mathrm{AB}}$ and (b) $\nu_{\mathrm{BC}}$ at $P^{*}=1.0$. The open symbols represent second-order wetting transitions.

When the temperature-dependent parameter $R$ is schematically described by the arrow shown in Figure 4, there are two wetting transitions predicted, as mentioned above. As the temperature increases from LCEP, the wetting transition, from nonwetting to partial wetting, is first order. Then the temperature is further increased to approach UCEP along the arrow, and the wetting transition, from partial wetting to complete wetting, is second order. The most intriguing phenomenon is that these two wetting transitions have different orders.

\section{Conclusion}

A density functional theory combined with the SAFT was successfully applied to describe the phase and wetting behaviors of ternary water + oil + amphiphile mixtures. The hydrophobicity parameter $R$ was proposed to represent the interaction strength between oil and amphiphile relative to that of water and amphiphile. Thus, a larger value of $R$ stands for a more hydrophobic amphiphile. An interfacial phase transition of nonwetting $\rightarrow$ partial wetting $\rightarrow$ complete wetting can be observed for the middle $\beta$ phase with increasing $R$. These results 
agree with the wetting behavior, as well as the phase behavior, of a water + tetradecane $+\mathrm{C}_{6} \mathrm{E}_{2}$ mixture $^{6}$ qualitatively.

\section{References and Notes}

(1) Chen, L.-J. Encylcopedia of Surface and Colloid Science; Hubbard, A., Ed.; Marcel Dekker: New York, 2002; pp 5630-5639.

(2) Antonow, G. N. J. Chim. Phys. Phys. Chim. Biol. 1907, 5, 372.

(3) Buff, F. P. Encyclopedia of Physics; Flugge, S., Ed.; Springer: Berlin, Germany, 1960; Vol. 10, Section 7, pp 298 and 299.

(4) Schick, M. Liquids at Interfaces (Les Houches Session XLVIII, 1988); Charvolin, J., Joanny, J. F., Zinn-Justin, J., Eds.; Elsevier: Amsterdam, The Netherlands, 1990; p 415.

(5) Aratono, M.; Kahlweit, M. J. Chem. Phys. 1991, 95, 8578

(6) Chen, L.-J.; Yan, W.-J. J. Chem. Phys. 1993, 98, 4830.

(7) Kahlweit, M.; Strey, R.; Busse, G. Phys. Rev. E 1993, 47, 4197.

(8) Chen, L.-J.; Hsu, M.-C. J. Chem. Phys. 1992, 97, 690.

(9) Chen, L.-J.; Hsu. M.-C.; Lin, S.-T.; Yang, S.-Y. J. Phys. Chem. 1995, 99, 4687.

(10) Chen, L.-J.; Yan, W.-J.; Hsu, M.-C.; Tyan, D.-L. J. Phys. Chem. 1994, 98, 1910.

(11) Chen, L.-J.; Lin, S.-Y.; Xyu, J.-W. J. Chem. Phys. 1996, 101, 225

(12) Kahlweit, M.; Busse, G. J. Phys. Chem. B 2000, 104, 4939.
(13) Chen, L.-J.; Chiu, C.-D.; Shau, F.-S.; Cheng, W.-J.; Wu, J.-G. J. Phys. Chem. B 2002, 106, 12782.

(14) Schick, M.; Shih, W.-H. Phys. Rev. Lett. 1987, 59, 1205.

(15) Gompper, G.; Schick, M. In Phase Transitions and Critical Phenomena; Domb, C., Lebowitz, J. L., Eds.; Academic: New York, 1994; Vol. 16.

(16) Talanquer, V.; Oxtoby, D. W. Faraday Discuss. 1999, 112, 91.

(17) Yeh, M.-C.; Chen, L.-J. J. Chem. Phys. 2003, 118, 8331.

(18) Jackson, G. Mol. Phys. 1991, 72, 1365.

(19) Carnahan, N. F.; Starling, K. E. J. Chem. Phys. 1969, 51, 635

(20) Rowlinson, J. S.; Swinton, F. L. Liquid and Liquids Mixtures, 3rd ed.; Butterworth: London, UK, 1982.

(21) Tarazona, P.; Telo da Gama, M. M.; Evans, R. Mol. Phys. 1983, $49,283$.

(22) Jackson, G.; Chapman, W. G.; Gubbins, K. E. Mol. Phys. 1988, $65,1$.

(23) Boublík, T. J. Chem. Phys. 1970, 53, 471.

(24) Kahlweit, M.; Strey, R. Angew. Chem., Int. Ed. Engl. 1985, 24, 654.

(25) Winsor, P. A. Trans. Faraday Soc. 1948, 44, 376.

(26) Evans, R. Adv. Phys. 1979, 28, 143.

(27) Tarazona, P.; Evans, R. Mol. Phys. 1983, 48, 799. 